\title{
L'ethos politique de Jean Lassalle à travers ses livres
}

Entre individualisation politique et représentation sociale

Jean Lassalle's ethos through his political books. Between political

individualization and social representation

El ethos político de Jean Lassalle a través de sus libros. Entre la

individualización política y la representación social

Clément Arambourou

URL : https://journals.openedition.org/mots/23484

DOI : $10.4000 /$ mots. 23484

ISSN : 1960-6001

Éditeur

ENS Éditions

Édition imprimée

Date de publication : 5 juillet 2018

Pagination : 131-147

ISSN : 0243-6450

Référence électronique

Clément Arambourou, "L'ethos politique de Jean Lassalle à travers ses livres », Mots. Les langages du politique [En ligne], 117 | 2018, mis en ligne le 05 juillet 2020, consulté le 23 avril 2022. URL : http:// journals.openedition.org/mots/23484; DOI : https://doi.org/10.4000/mots.23484 


\title{
L'ethos politique de Jean Lassalle à travers ses livres
}

\author{
Entre individualisation politique \\ et représentation sociale
}

Avant d'apparaître nationalement comme un des onze candidats à l'élection présidentielle de 2017 , Jean Lassalle (voir encadré ci-dessous) avait déjà fait l'objet d'une couverture médiatique certaine - attestée par le recensement de ses passages à la télévision (Leroux, Riutort, 2013) -, et cela grâce à son tour de France à pied effectué du 10 avril au 11 décembre 2013, sa grève de la faim menée du 7 mars au 14 avril 2006 ou son chant béarnais entonné le 3 juin 2003 dans l'hémicycle de l'Assemblée nationale.

Les mises en scène des actions politiques de ce député ne passent pas seulement par la télévision, mais aussi par le livre politique (Neveu, 1992; Le Bart, 1998a et 2012). J. Lassalle est aujourd'hui l'auteur de cinq ouvrages. En 2008, il publie La parole donnée (désormais $L P D$ ), ouvrage à forte tonalité autobiographique. En 2011 sort Le retour du citoyen (désormais $L R C$ ), qui mêle récit de son activité d'élu et réflexions politiques. À la rencontre des Français (désormais $A L R F$ ), publié en 2015, est un opus spécifiquement consacré à sa marche à travers la France. Avec sa candidature à l'élection présidentielle, la bibliographie de J. Lassalle s'enrichit de deux nouveaux ouvrages. Il s'agit d'Un bergerà l'Élysée en 2016 puis de Résistons! en 2017, ouvrages plus programmatiques que les précédents écrits de ce député. Les publications de J. Lassalle sont toutes associées à une phase ascendante de sa carrière politique. Les trois premiers ouvrages sont censés lui permettre de "s'imposer comme entrepreneur politique individuel» en compensant un «isolement» (Le Bart, 2012, p.114-115); J. Lassalle appartient en effet au Mouvement démocrate (MoDem), parti politique dont la représentation à l'Assemblée nationale est très réduite entre 2007 et 2017 (trois puis deux députés). Ses deux derniers ouvrages visent plutôt à « [l']imposer comme leader » (Le Bart, 2012, p. 114). Après avoir quitté le MoDem en 2016, il est candidat à l'élection présidentielle de 2017. Il tente alors de rassembler autour de

Université de Bordeaux, centre Émile Durkheim (UMR 5116) arambourouclement@yahoo.fr 
sa personne et de son nouveau mouvement, baptisé Résistons!, présenté dans le livre du même nom. Les publications politiques de ce député béarnais sont donc étroitement associées à son émergence comme acteur politique national.

Un livre politique produit un ethos permettant de se «poser en personnalité politique légitime » note Ruth Amossy (Amossy, 2014, p. 24). Cette notion d'ethos est notamment mobilisée par les études politiques menées en sciences du langage (Charaudeau, 2005). La science politique utilise plus fréquemment la notion d'identité politique qui conçoit l'image d'une personnalité politique comme le résultat d'un processus d'interactions entre présentation par autrui (alliés, adversaires, commentateurs de la vie politique, etc.) et présentation de soi (Lefebvre, 2004). En tant qu'image donnée de soi à travers les discours, l'ethos est un élément de l'identité politique. Son étude consiste à isoler des stratégies plutôt qu'à considérer des effets performatifs (Amossy, 2014) - comme la production d'une «identité stratégique» (Collovald, 1988) - difficilement objectivables. Toute personnalité créditée d'un minimum de représentation politique est « une marque politique, c'est-à-dire non seulement un entrepreneur politique individuel mais l'expression d'une équipe et d'un certain nombre de valeurs symboliques» (Sawicki, 1994, p. 37). Peu importe donc que «[l]es hommes politiques [...] n'écrivent pas [...] forcément tout ce qu'ils publient» (Le Bart, 1998a, p. 77) et qu'ils recourent, comme ailleurs dans l'exercice de leurs rôles politiques, au «travail» de standardisation «des communicateurs et consultants» (Lagroye, 2003, p.55). En effet, ce sont les stratégies de présentation par la publication qui sont intéressantes et ces dernières ont toutes les chances d'avoir été validées par l'intéressé. Pour le politiste, la biographie est un «enjeu : elle est l'occasion et le moyen que saisit l'acteur pour renvoyer aux autres une certaine image de lui-même» (Joana, 1994, p. 95).

Mais « [l]'ethos ne renvoie pas uniquement à un orateur individuel qui projette une image singulière de sa personne. Le “je” peut se faire le porte-parole d'un groupe» (Amossy, 2014, p. 23). La notion d'ethos permet donc l'étude des pratiques de la représentation politique (Mazeaud éd., 2014). Elle est également utilisée en sociologie des professions afin de désigner les manières d'exercervalorisées par les professionnels (Fusulier, 2011). En tant que produit d'une division du travail, le champ politique distingue des professionnels de la représentation politique (Bourdieu, 2001a; Gaxie, 2003) dont l'activité de représentation du monde social se double d'un travail concurrentiel de légitimation personnelle auquel participent les ethos produits. Reprendre la perspective de la sociologie des champs conjointement avec l'usage de la notion d'ethos (Amossy, 2011) et tenir compte du travail de représentation politique, ce n'est pas seulement mettre en relation l'ethos visé par J. Lassalle avec la vision du monde qu'il manifeste, c'est aussi lier cet ethos avec l'évolution des critères de légitimité du champ politique en général et de ceux qui concernent l'écriture en politique en particulier. 
Pour Christian Le Bart (2012), le livre politique est jusqu'aux années 1970 réservé aux présidentiables pour qui l'image d'homme de lettres est un quasiimpératif identitaire. À partir des années 1980, organisations partisanes et institutions politiques perdent en légitimité. La composition du capital politique valorisé s'enrichit en ressources individuelles. Dès lors, le livre politique est un objet qui se banalise et le régime discursif de la singularité se diffuse à travers l'imposition d'une présidentiabilité ordinaire; le face-à-face entre un homme (ou une femme) et un peuple est un récit largement repris. Les professionnels de la politique s'adressent directement aux lecteurs-électeurs et se mettent personnellement et explicitement en scène. À l'ère de l'ego-politique, d' «affaire d'institutions [...] s'incarnant dans des personnes», la politique " est devenue une affaire d'individus [...] s'appuyant, mais moins systématiquement qu'avant, sur des institutions» (Le Bart, 2013, p. 20). En accord avec cette ego-politique, J. Lassalle revendique un caractère atypique. Il se présente ainsi dans le cadre du scrutin présidentiel : «Vous le savez, j’ai toujours été différent et c'est cette différence que, je crois, vous aimez chez moi. Soyez bien certains, mes chers amis, que cette différence je l'emporterai avec moi à l'Élysée» (Résistons!, p. 79).

Les règles du champ politique structurent mais ne contraignent pas intégralement la production des discours politiques (Le Bart, 1998b et 2003a). Ainsi, dans le cas des livres publiés par J. Lassalle, la question de la reprise des principes de légitimation utilisés dans la vie politique contemporaine en général et dans les publications politiques en particulier révèle une variation singulière autour de cette ego-politique. Pour Christian Le Bart, l'individualisation du champ politique conduit ses acteurs à renoncer, au moins en partie, à incarner les «groupes sociaux» (Le Bart, 2013, p. 219) pour mettre en sens les expériences sociales à l'aide des émotions (Faure, Négrier éd., 2017). Or, dans les récits proposés par J. Lassalle, la production d'une personnalité dotée de qualités personnelles remarquables par le recours aux affects se réfère bien souvent à des catégories et des divisions du monde social. C'est ce que nous mettrons en évidence, en montrant que cette variation autour de l'ego-politique n'est pas sans lien avec les propriétés sociologiques singulières de cet homme politique.

\section{Présentation du parcours politique de J. Lassalle}

Né le 3 mai 1955 à Lourdios-Ichère, titulaire d'un brevet de technicien agricole, J. Lassalle est élu maire de son village natal en 1977. En 1982, il devient conseillergénéral du canton d'Accous et siège comme conseillergénéral indépendant. En 1988, c'est désormais sous les couleurs du Centre des démocrates sociaux (CDS), parti membre de l'Union pour la démocratie française (UDF), qu'il se présente comme suppléant du député de la quatrième circonscription des Pyrénées-Atlantiques (Michel Inchauspé, Rassemblement pour la République) et comme conseiller général. Au CDS, il rejoint des leaders 
politiques locaux tels Henri Grenet (alors maire de Bayonne et président du conseil général) et Didier Borotra (alors vice-président du conseil général et vice-président du conseil régional), ainsi que des élus plus jeunes et en devenir, à savoir Jean-Jacques Lasserre (actuel président du département des Pyrénées-Atlantiques) et François Bayrou (futur ministre et futur présidentiable). En 1991, il devient vice-président du conseil général des Pyrénées-Atlantiques. Il est élu député titulaire en 2002. Il adhère au MoDem l'année de sa fondation par F. Bayrou, en 2007. J. Lassalle mène ensuite la liste Aquitaine de ce parti lors des élections régionales de 2010, liste qui se qualifie pour le second tour à l'issue duquel elle arrive en troisième position. La même année, il accède au poste de vice-président du MoDem et obtient le portefeuille «Égalité des territoires, identités locales, services publics» au sein du shadow cabinet de F. Bayrou. Alors que le président du MoDem perd son siège, J. Lassalle parvient à être réélu lors du scrutin législatif de 2012 - il bénéficie pour sa part du soutien de l'Union pour un mouvement populaire (UMP) en raison de son vote pour Nicolas Sarkozy au second tour de l'élection présidentielle de 2012 (au contraire du choix fait par F. Bayrou). J. Lassalle est réélu maire de Lourdios-Ichère en 2014. II ne se représente pas au scrutin départemental de 2015 marqué par la fusion des cantons et l'instauration d'un ticket paritaire. La même année, la tête de la liste d'union de la droite et du centre en lice sur la grande région Aquitaine-Limousin-Poitou-Charentes lui échappe et revient à une proche d'Alain Juppé, Virginie Calmels. En mars 2016, critiquant le soutien que F. Bayrou apporte au projet de candidature présidentielle d'A. Juppé, il annonce sa volonté de se présenter à l'élection présidentielle de 2017. Malgré l'absence de soutien d'une véritable organisation partisane - F. Bayrou soutiendra la candidature d’Emmanuel Macron à la suite de la défaite d'A. Juppé à l'élection primaire organisée par Les Républicains -, il obtient les 500 signatures d'élus nécessaires pour figurer parmi les onze candidats en lice. Avec 1,21\% des suffrages exprimés, J. Lassalle arrive en septième position au soir du 23 avril 2017. Le 18 juin suivant, à l'occasion du second tour des élections législatives, il est réélu député des Pyrénées-Atlantiques face à un candidat appartenant à La République en marche du nouveau président de la République

E. Macron; nombreux furent pourtant les parlementaires sortants à faire les frais du succès électoral de cette nouvelle formation politique.

\section{Fils du peuple, distinction politique et identification paysanne}

«[I]ssu du peuple, peuple lui-même, au service du peuple, l'élu en sera la voix au Parlement parce qu'il en est le fils légitime et méritant» écrit Bernard Pudal (Pudal, 1989, p. 213) à propos des biographies et portraits des députés commu- 
nistes élus en 1936. J. Lassalle est historiquement et idéologiquement éloigné des élus du Front populaire. Il revendique pourtant lui aussi une filiation populaire :

La ferme de mes parents est située au fond de la vallée d'Aspe, en lisière du petit village de Lourdios-Ichère, sur un versant dont la pente est si forte qu'elle est aussi difficile à arpenter qu'à travailler. On y accède par un chemin boueux, étroit et malcommode. Pour héberger nos bêtes, quatre-vingts brebis, une quinzaine de vaches, les cochons, les poules, l'âne, sept petites granges s'échelonnaient alors sur le flanc de la montagne. En période d'hiver, chaque jour il fallait en accomplir deux fois le tour pour donner à boire et à manger aux animaux, les faire sortir, changer leurs litières, vider le fumier. Le travail était démesuré pour un rapport financier plus que modeste. (LPD, p.9-10)

La manifestation de cette appartenance populaire passe encore par le récit de son rapport difficile à l'institution scolaire. Pour Pierre Bourdieu et JeanClaude Passeron, l'héritage culturel scolairement dévalorisé des enfants des classes populaires permet de rendre compte de leur élimination statistique au fur et à mesure que l'on progresse dans les hiérarchies du système d'enseignement (Bourdieu, Passeron, 1964). Les deux sociologues considèrent également l'effet d'autres handicaps tels que la résidence en milieu rural. Dans un travail ethnographique consacré au célibat des hommes paysans en Béarn et mené au début des années 1960, P. Bourdieu observe que, depuis peu, les filles des familles paysannes béarnaises poursuivent plus souvent leurs études que les garçons (Bourdieu, 2001b). Au niveau national et à partir de 1971, les filles deviennent majoritaires parmi les candidates et les candidats reçus au baccalauréat (Baudelot, Establet, 1992). J. Lassalle appartient donc à une cohorte où appartenances paysanne et masculine sont des désavantages scolaires. J. Lassalle met en récit cette expérience paysanne de la domination scolaire. Ainsi, il raconte qu'avant d'entrer à l'école, il ne parlait que le béarnais et le cheso ( $L P D$, p. 23), d'où un malaise ressenti au contact de la culture scolaire, malaise renforcé par la confrontation à certains usages du corps, comme ceux que nécessitent les jeux de balle - J. Lassalle parle de son inaptitude au ballon et dit se sentir "gauche» ( $L P D$, p. 23). À l'époque de l'enquête ethnographique de P. Bourdieu, l'équipe de rugby du village était " presque exclusivement» composée «des “citadins” du bourg» (Bourdieu, 2001b, p.115) qui disposaient seuls des compétences sportives nécessaires. J. Lassalle évoque donc un corps que P. Bourdieu qualifie d' "empaysanné», expérience qui est le lot des jeunes hommes béarnais confrontés à une école qui constitue alors «l'instrument principal de la domination symbolique du monde citadin »(Bourdieu, 2001b, p. 237).

Professionnel de la politique, J. Lassalle siège au sein d'une Assemblée nationale sur les bancs de laquelle $76 \%$ des élus ont un diplôme supérieur à Bac+2 (Rouban, 2011). En tant que titulaire d'un brevet de technicien agricole, J. Lassalle appartient aux $24 \%$ restants. Or, les travaux de sociologie politique s'accordent sur le fait que le capital culturel est un facteur principal 
d'explication des inégalités d'accès à la parole et au porte-parolat politiques (Gaxie, 1978 ; Cotta, Best éd., 2007). La mise en récit des expériences scolaires de J. Lassalle manifeste une relation d'identité avec les classes populaires rurales et une différenciation vis-à-vis du personnel politique national. Néanmoins, et malgré ce passé scolaire difficile, J. Lassalle se veut à l'image de son grand-père paternel dont il est l'homonyme :

Jean Lassalle était un homme relativement cultivé, non pas qu'il ait été à l'école mais parce qu'il avait appris de lui-même. Très proche des bêtes, il l'était aussi des hommes qu'il comprenait parfaitement. Il fut un des tout premiers syndicalistes. C'était un homme auprès duquel on venait chercher parfois de très loin conseil et recours. Il était par ailleurs sourcier et avait fait creuser de nombreux puits. (LPD, p.14)

Comme les députés et dirigeants communistes du Front populaire étudiés par B. Pudal (Pudal, 1989), l'élu béarnais met en avant ses apprentissages d'autodidacte. C'est, dit-il, grâce au goût du travail hérité de son père et face aux nécessités pratiques qu'il acquiert des savoirs dont l'abstraction pouvait jadis le dépasser. Il souligne ainsi ses difficultés d'élève du secondaire confronté aux équations mathématiques. Mais il dit parvenir, dans le cadre de son activité professionnelle, à surmonter ses réticences en puisant dans son tempérament de travailleur acharné. J. Lassalle raconte ainsi qu'il a pris, jeune adulte, la tête de son propre cabinet d'études techniques et agricoles. Il décroche alors un marché de construction de lignes électriques, ce qui n'est pas sans lui poser de sérieux problèmes :

Sauf que je n'étais pas très éclairé quant à la façon de construire des lignes électriques! Je me suis donc remis à bûcher, j'ai écouté les uns, les autres, consulté des copains d'EDF qui m'ont tous mis en garde : «Tu es fou! On y a passé des années pour apprendre! Tu n'y arriveras jamais!». L'un d'eux, Charly, un ami très proche, entreprit même de me donner des cours du soir. ( $L P D$, p. 85)

Je crois que je n'ai jamais autant travaillé que durant cette période-là. Le jour, j'enchaînais les réunions du conseil général et les visites de terrain, puis toute la nuit je plongeais dans les calculs abscons des pertes de charge et d'évaluation du poids de la neige sur les fils électriques. (LPD, p. 86)

À travers son ethos biographique, J. Lassalle propose une vision d'un monde social où le «mérite scolaire », relativisé et non pas dénié, est complété par cet «autre mérite», le «mérite professionnel» (Duru-Bellat, Tenret, 2009, p. 251). Dans cet idéal méritocratique que J. Lassalle entend incarner, les verdicts scolaires sont loin d'être des fatalités pour ceux qui savent redoubler d'efforts. La prise de responsabilités publiques est une voie qui leur est alors ouverte. J. Lassalle est élu maire en 1977 puis conseiller général en 1982. Il rend compte de son engagement politique par la volonté de représenter et de défendre sa catégorie sociale d'origine, catégorie modeste, injustement stigmatisée et privée de parole politique. Le «je » se mue ainsi en «nous», «l'ensemble des paysans»: 
Dès ces années [d'enfance] j'ai su que nous ne nous réduisions pas à ce que nous semblions être; nous, c'est-à-dire ma famille, mais aussi les gens des âpres vallées montagnardes et au-delà l'ensemble des paysans. Cela s'apparentait à une promesse, certes informulée, mais dont les termes n'en étaient pas moins forts. Nous n'appartenions pas au cercle de ceux qui avaient droit de cité, cependant il faudrait bien que ces gens-là nous entendent et nous écoutent. (LPD, p. 37)

Si l'ouvriérisme est bien "un habitus ouvrier revendiqué, brandi », une "classe incorporée faite conviction » et une "voie de passage quasi obligée qui mène de l'indignité sociale à la dignité "ouvrière” " (Pudal, 1989, p.133134), J. Lassalle est alors un représentant du «paysanisme» (Lagrave, 2009, p. 56). Comme certains députés poujadistes (Collovald, 1989; Offerlé éd., 2017) et plus généralement les outsiders du champ politique étudiés par Christine Guionnet, J. Lassalle prétend « mieux représenter le corps social dans la mesure où [il] posséderait des ressources singulières, liées à des caractéristiques plus sociales que politiques » (Guionnet, 2002, p.127). Mais l'ethos de représentant produit par les livres politiques de J. Lassalle ne fait pas seulement référence à une origine paysanne, il renvoie également à un idéal artisanal.

\section{Un artisan en politique}

J. Lassalle souhaite « réhabiliter par une grande campagne nationale les métiers qui font honneur à l'intelligence de la main, courroie de transmission directement reliée au cœur, et qui impose son talent à la matière» ( $L R C$, p. 107). En cohérence avec son programme, cet homme se met en scène en train d'accomplir des travaux manuels. Quittant à l'occasion son habit de député, il participe alors à l'entretien de l'exploitation familiale :

La sueur coule en perles fines le long de mon corps. Je ressens des picotements autour du cou et mes yeux s'embrument. Je me redresse pour éponger mon front avec un mouchoir taché par un peu de terre. La tête me tourne, comme si le sol se dérobait soudain sous mes pieds. J'ai posé la tronçonneuse en équilibre instable sur la pente [...]. Je contemple avec satisfaction les troncs que je viens de scier, éparpillés entre les ronces gelées.

[...] Je ressens maintenant plus nettement les premières irritations du froid; la seule attitude raisonnable consiste à reprendre l'ouvrage. Le vieux chêne subit donc son deuxième assaut de la matinée tandis que le soleil commence à monter. [...] Dans le feu de l'action, et tout en restant aussi prudent et méthodique que l'exige pareil exercice, je ressens les douleurs qui remontent de mon dos endolori, mes jambes qui tirent un peu, tandis que mon regard parfois vacille, surtout lorsque je me baisse un peu rapidement.

[...] Il faut bien observer le sens des branches tordues, entre le tronc et le sol; une mauvaise appréciation peut vous valoir un accident, tout comme une branche brusquement libérée vous décapiter ou vous ouvrir la poitrine. Je redouble d'attention. Le travail des muscles, retrouvant peu à peu force et souplesse, me permet de 
récupérer progressivement de l'assurance. [...] Je suis plutôt fier de moi et me félicite en mon for intérieur : "Tu n'es pas si rouillé, pour la vie que tu mènes. Et sans entraînement, abattre un tel labeur en trois heures, ma foi, ce n'est pas si mal!»

[...] C'est décidément toujours la même chose pour moi, lorsque je reprends brutalement, à mes moments perdus ou pendant les vacances, ce travail physique. Ce n'est pas comme mon frère Julien, rompu à cette vie rude, qui a choisi à ses 18 ans de s'occuper de la redoutable exploitation familiale [...]. Une grande partie des habitants de Lourdios-Ichère ont comme lui, à la fin des années 1970, fait ce choix difficile et admirable de rester sur leurs terres. Je viens l'espace d'une froide matinée, de tutoyer leur quotidien. (LRC, p.12-14)

Tout d'abord, ce récit parle d'une mobilité sociale ascendante (Naudet, 2012). J. Lassalle met en scène une réduction de la tension entre milieu d'origine et groupe d'appartenance, et témoigne d'une humble admiration pour les qualités morales et physiques nécessaires à l'accomplissement du travail paysan. Ces lignes révèlent une forme de «nostalgie chevaleresque » (Le Bart, 2003b) pour une existence plus authentique. La reconnaissance témoignée ici à ceux qui ont opté pour une activité paysanne constitue une stratégie d'atténuation de la distance entre représentant et représentés typique d'une rhétorique de la proximité (Le Bart, Lefebvre éd., 2005). Ensuite, la narration de ce travail de bûcheronnage accompli par J. Lassalle s'inscrit parfaitement dans la division sexuée des tâches agricoles (Bourdieu, 1980). La tronçonneuse est un substitut moderne de cet outil-arme qu'est la hache, "ornement masculin "total" " (Tabet, 1979, p. 39). Ici, et comme dans le coupage des branches chez les Bemba, «[l]e travail [masculin] est [...] vu comme une action "héroïque", guerrière, courageuse, comme une compétition ou un jeu » (Tabet, 1979, p. 40).

Le récit de ces activités extra-parlementaires s'inscrit dans une prise de distance générale vis-à-vis des manières avec lesquelles le personnel politique en place accomplirait son travail de représentation :

Comment faire comprendre que la politique doit plus que jamais se nourrir d'émotion et de passion, alors que beaucoup pensent aujourd'hui qu'elle ne se résume plus qu'à de la technique? [...] Si la certitude est un peu l'opium du technocrate, la recherche et l'enthousiasme doivent être celui de l'élu. Pour l'heure, nous sommes coupés des citoyens, lesquels ne savent pas, ne savent plus, ce que nous faisons. (LPD, p. 352)

Oui, la politique est un art et, comme dans tout art, il faut des artistes. Il me semble que nous en manquons un peu aujourd'hui dans bien des domaines. Le nôtre n'y échappe pas. C'est pourquoi n'hésitez pas : engagez-vous! L'art, c'est la conjonction magique du cœur, de l'esprit et de l'âme. Si nous les ramenons à la politique, nous aurons de beaux jours devant nous. (LPD, p. 353)

Et si la politique redevenait un art? Pourquoi ne redeviendrait-elle pas l'expression du rêve, de l'idéal, du beau, oserais-je : du sublime? Oui. Le chemin est long mais nos esprits sont déjà en route. ( $A L R F$, p.308) 
La technocratie est une catégorie politique de dénonciation largement mobilisée (Dubois, Dulong éd., 1999). Cette forme de bureaucratie s'oppose ici à la créativité et à la liberté de l'artiste. Les propriétés émotionnelles que J. Lassalle met en discours sont donc des outils de stigmatisation du rôle de technocrate caractérisé par la rationalité, la rigidité et la froideur, bref, par tout ce qui évoque la mise à distance des êtres, des choses et des émotions. La critique de J. Lassalle vise plus largement le «monstre technocratique», ledit «jacobinisme» français et sa «bureaucratie féroce» frappant du «sceau de la mort» les «derniers paysans et les ultimes bergers» (LPD, p. 51-52). Cette "technocratie» se serait de plus désormais mis «en tête de mettre un terme à l'une des originalités et des constantes auxquelles notre pays est attaché depuis des centaines d'années : la relation intime entre l'homme et le territoire » ( $A L R F, \mathrm{p} .37)$, et cela à travers différentes réformes territoriales. Ce jugement sévère porté sur les institutions bureaucratiques n'épargne pas les organisations partisanes contre lesquelles ce député entend représenter le peuple grâce à sa relative identification aux représentés d'une part, et en vertu d'une personnalité particulière d'autre part :

[L]es grands partis ont mis des colliers électroniques à leurs députés comme aux ours [des Pyrénées]. [...] Vous les croyez libres? Vous vous trompez. Ils sont aux ordres. [...] [S]i vous voulez un député qui vous ressemble, qui ne vote pas systématiquement pour ou contre, qui examine les textes en fonction de l'intérêt des citoyens, votez pour moi. Il ne faut pas envoyer des moutons à l'Assemblée mais des bergers. (LPD, p. 314-315)

Si je suis réélu, disais-je aux électeurs, je continuerai comme j'ai toujours fait. C'est-à-dire que je n'en ferai qu'à ma tête, ce qui est la meilleure façon de vous représenter. Mais attention! En faire à sa tête, ce n'est pas faire n'importe quoi. C'est agir comme son intelligence et ses convictions vous le commandent. (LPD, p. 308-309)

J. Lassalle développe une critique "populiste» (Kriesi, 2014) qui cible les partis et les élites en place et promeut un nouveau lien entre leaders politiques et citoyens. Cette critique populiste prend la forme d'une «critique artiste» (Boltanski, Chiapello, 2011), celle d'une protestation contre la perte d'autonomie et d'authenticité. C'est, plus précisément, une critique d'artisan. En effet, l'ethos politicien de J. Lassalle est analogue à l'ethos professionnel de l'apprenti que décrit Bernard Zarca, ce «fils d'artisan, d'ouvrier de l'artisanat, d'exploitant agricole traditionnel» qui «vient d'un milieu social qui cultive des valeurs de travail et d'effort», dispositions qui permettent d'acquérir « une manière d'être, un idéal : la réussite professionnelle par l'accès à la maîtrise du métier et son exercice indépendant» (Zarca, 1988, p. 250). La profession exercée par J. Lassalle avant qu'il ne se professionnalise en politique et sa manière de représenter en politique ont pour point commun cette valorisation de l'indépendance. Dans l'idéal artisanal le plus exigeant, le corps et le travail manuel 
sont des voies d'accès au sentiment «esthétique » (Zarca, p. 253), sentiment qui doit selon l'élu béarnais nourrir le travail de représentation politique. Les artisans, explique encore B. Zarca, ces «hommes de métier», en «maîtrisant le faire », s'opposent aux «puissants [qui] ont le quasi-monopole de la parole » et défendent ainsi leurs positions, sociale et masculine (Zarca, 1993, p. 88). C'est bien ces puissants sourds aux voix du peuple que J. Lassalle entend dénoncer et concurrencer. Cette référence à un idéal artisanal implicite ne peut se comprendre que si l'on garde à l'esprit le fait que «l'artisanat est la plus populaire des classes moyennes» (Zarca, 1993, p. 55). L'ethos artisanal offre donc un triple profit d'affichage : relative réussite sociale, identification à l'entrepreneuriat - un des référentiels principaux des «mondes agricoles » (Hervieu, 2010) - et appartenance populaire.

Ces valeurs d'effort associées aux classes populaires, au milieu rural et à l'idéal artisanal, J. Lassalle les exprime encore à travers un ethos de combattant en politique.

\section{Un combattant en politique, une masculinité pacifiée}

Nous avons déjà relevé la masculinité de l'ethos produit par les ouvrages politiques de J. Lassalle. Cette dernière apparaît notamment et nettement dans la mise en scène d'une image de combattant en politique censée être révélée par les performances de J. Lassalle (chant à l'Assemblée nationale, grève de la faim dans la salle des quatre colonnes et marche à travers la France). C'est une capacité à «faire face » (Bourdieu, 2002) qui se donne tout d'abord à voir dans la participation à l'abattage d'un cochon, lequel constitue à la fois un travail agricole masculin ${ }^{1}$ et un «rite d'institution » (Bourdieu, 2001a) :

L'une des émotions les plus fortes de ma vie fut la première mise à mort à laquelle je me livrai sur un cochon. Je venais d'avoir 15 ans et, ce jour-là, tout le monde ou presque se ligua contre moi pour décréter qu'il me faudrait le saigner et l'éventrer. Fidèles à la tradition, nous prîmes un solide casse-croûte, suivi du café, et du pousse-café de circonstance. Puis nous, les hommes, nous nous levâmes pour aller saisir l'animal de plus de 200 kilos, tandis que les femmes s'affairaient à l'intérieur. [...]

De ma main droite, j'enserrai ma main gauche contre sa gueule en l'enlaçant dans la cordelette. Il n'était plus que bave et fureur, ses yeux me jetaient des éclairs

1. En Béarn, ce rite d'institution est particulièrement marqué par la division des sexes. P. Bourdieu raconte : «J'ai ainsi le souvenir que, dans mon enfance, les hommes, voisins et amis, qui avaient tué le cochon le matin, dans un bref déploiement, toujours un peu ostentatoire, de violence - cris de l'animal qui s'enfuit, grands couteaux, sang versé, etc. - restaient pendant tout l'après-midi, et parfois jusqu'au lendemain, à battre tranquillement les cartes, à peine interrompus pour soulever un chaudron trop lourd, pendant que les femmes de la maison s'affairaient de tous côtés pour préparer les boudins, les saucisses, les saucissons et les pâtés. » (Bourdieu, 2002, p.50). 
de mort. Je fus surpris, l'espace d'un instant, par l'étrange sérénité qui m'avait soudain gagné au moment même où j'étais animé d'une froide détermination. Je pris l'énorme couteau que l'on me tendait et, comme je l'avais déjà vu faire tant de fois, les larmes aux yeux, je commençais à tâter le cou tendu de la bête pour bien percevoir l'endroit où j'allais enfoncer la lame jusqu'à la garde. Pour un coup d'essai, ce fut un coup de maître et j'atteignis même, fait rarissime, le cœur. Le sang gicla violemment de sa gorge percée tandis que maman s'était mise en position pour le recueillir. Les hommes, d'un seul coup libérés, tout en restant très concentrés, saluèrent aussitôt la qualité de mon coup de couteau et l'animal mourut très vite dans un terrible soubresaut et râle violent. Mes membres s'étaient remis à trembler tandis que je libérais ma main recouverte de son sang. D'où m'était venu ce brusque accès de force sauvage qui, annihilant complètement mes sentiments, m'avait rendu à ce point féroce?

Mon père, qui n'avait pas participé à la scène, s'approcha de moi très doucement et me félicita : «Tu sais, je suis fier. Ce que tu viens de faire, jamais je n'ai pu le faire.» (LPD, p. 42-45)

Dans la continuité de cette présentation de soi, Le retour du citoyen se termine par une reproduction in extenso de la traduction du poème If de Rudyard Kipling par André Maurois (Tu seras un homme, mon fils).

La capacité de violence de J. Lassalle ne s’applique jamais physiquement à un autre être humain ; en politique, l'ethos viril du combat se doit d'être pacifié (Elias, Dunning, 1994). Il est alors un outil de stylisation des relations politiques au sein desquelles J. Lassalle joue un rôle de chevalier. Dans le cadre d'une rhétorique de la nostalgie chevaleresque (Le Bart, 2003b), ce rôle guerrier est parfaitement compatible avec la manifestation d'un ethos paysan et artisanal; en effet, cette rhétorique se nourrit de tous les récits qui permettent de se distancier des faux-semblants d'un monde politique aseptisé. Ainsi, J. Lassalle évoque la réaction d'Alain Rousset au moment de sa grève de la faim. II rappelle que le président de la région Aquitaine s’interrogeait alors publiquement sur la possibilité que le député béarnais en garde des séquelles psychiatriques. C'est la nécessité de répondre à cet affront qui justifie dans le récit la candidature de J. Lassalle lors du scrutin régional de 2010 :

Son acte m'avait alors fait l'effet d'un gant que j'aurais reçu en pleine figure lors d'un bal à la cour du XvIII siècle. Les duels armés n'existant plus, je savais dans mon for intérieur que je ne retrouverais la paix que procure la réparation de l'outrage qu'après l'avoir combattu politiquement en combat singulier. (LRC, p. 61-62)

Historiquement, les duels s'insèrent dans une «culture de l'honneur masculin» issue d' "une synthèse d'éléments nobles et bourgeois» (Nye, 1994). L'imaginaire du duel est donc typique de la nostalgie chevaleresque animant les individus insérés dans des rapports politiques largement pacifiés. Le récit du chant béarnais entonné par J. Lassalle dans l'enceinte de l'Assemblée nationale présente lui aussi un affrontement pacifié. C'est pour protester contre la 
suppression d'une gendarmerie installée sur son territoire d'élection que J. Lassalle décide, en 2003, d'interrompre le ministre de l'Intérieur, N. Sarkozy, au moment où ce dernier répond à une question au gouvernement :

J'entonnais le chant, tout en prenant soin de rester le plus droit et le plus digne possible parce que, même dans ce moment atypique de ma vie parlementaire, je n'oubliais pas que je représentais mes électeurs, et au-delà les citoyens de la nation entière. Le chant se déployait maintenant sans heurts, "Aqueros mountagnos que tan hautes soun, m'empéchon de bede, mas amous oun soun... » [...]. Tout en bas de l'hémicycle, au fond en somme, Nicolas Sarkozy tentait de répondre à la question qui lui avait été posée. Il ramait, le pauvre. Puis il commença à bafouiller, lança des gestes désordonnés des bras tandis que ses coups de pied tapaient dans la balustrade. Puis il finit par s'immobiliser, me regardant, le visage sans expression. L'espace d'un instant je vis bien qu'il avait été déchu de sa position favorite. Il n'était plus le centre de l'attention générale et sa légendaire maîtrise des situations avait craqué sous les coups d'un énervement manifeste.

Quant à moi, j'avais trouvé le bon ton et le bon rythme, tout allait bien. Je haussai encore le son de ma voix, parce que l'hémicycle est vaste et que je n'avais pas de micro. Quant au président de l'Assemblée, Jean-Louis Debré, il hurlait dans son micro [...].

Je tenais les deux puissants dans mon viseur et je continuais à chanter, plus conscient que jamais de représenter tous les oubliés dont on décide du sort sans même prendre le soin de les entendre. (LPD, p. 194-196)

Cet affront fait à N. Sarkozy et J-L. Debré joue sur une division entre les élites (les «puissants») et le peuple (les «oubliés») symbolisée par une opposition des attitudes corporelles. D'un côté, J. Lassalle, voix forte, corps droit, port fier et digne; il reste imperturbable. De l'autre côté, J-L. Debré et, surtout, N. Sarkozy aux mouvements désordonnés et inefficaces qui trahissent une colère impuissante. Ceux qui maîtrisent l'agenda et les décisions politiques sont ici incapables de maîtriser leurs corps et leurs émotions, corps et émotions qui, chez J. Lassalle, restent contrôlés - la maîtrise (des choses mais aussi de soi) est un invariant anthropologique de définition de la masculinité (Singly, 2013). Cette maîtrise et cette combativité se veulent au service d'un rassemblement excluant toute option violente :

Je suis un combattant qui n'aime pas les conflits. Toujours j'ai voulu apaiser, arranger, parler, me situer dans le débat et non dans l'opposition. (LPD, p. 20)

Marcher pour entendre, marcher pour comprendre. On maîtrise une situation tant que le sang n'a pas coulé, et je me disais qu'il n'était plus possible, en 2013, de condamner une nouvelle génération à être saignée dans une impasse redoutable. Il fallait rechercher les prémices d'un nouveau paradigme politique, chercher ce qui pouvait de nouveau unir les Français, plutôt que de pointer ce qui les divisait. Et, par là même, tenter d'entrevoir des issues et de dessiner des réponses. (ALRF, p. 43) 
Le courage pacificateur de J. Lassalle se donne encore à voir à l'occasion du récit d'une étape de sa marche lors de laquelle il raconte faire obstacle à une fusillade. Dans le récit de J. Lassalle, un cafetier souhaite abattre un caïd du quartier où son commerce est installé, et cela afin de venger son fils gravement blessé par ce dernier. J. Lassalle qui se trouvait de passage dans le cadre de son tour de France raconte avoir empêché le drame en préparation en saisissant courageusement le fusil d'assaut des mains du commerçant :

Je ne perdais pas le patron des yeux. Il était toujours occupé avec son fusil car, fort heureusement, le chargeur ne semblait pas s'enclencher. Il voulait manifestement en finir au plus vite. Soudain, je perçus que sa main tremblait légèrement. Je lui arrachai l'arme et la lançai au gars qui venait d'entrer. (ALRF, p. 183-184)

Dans les récits de J. Lassalle, les épreuves qu'il traverse, en plus de révéler ses rares qualités physiques et morales, se voient nimbées d'un certain mysticisme. Cela est notable dans le récit de son refus de s'alimenter pendant plusieurs dizaines de jours :

Je m'étais certes allégé de 20 kilos, ce qui soignait ma ligne mais m'affaiblissait au point de ne presque plus pouvoir marcher. Les médecins qui venaient m'examiner étaient surpris de ma résistance et d'un état général plutôt satisfaisant. (LPD, p. 240-241)

On me brancha donc un goutte-à-goutte qui m'instillait seulement de l'eau salée. En ce jour de Pâques, je me surpris à sourire en pensant : «l'espère que le Christ ne va pas m'en vouloir» car lui s'était alimenté au quarantième jour! (LPD, p. 249)

La marche est une autre épreuve quasi biblique qui le prédispose à une imputation charismatique (Weber, 1995), si l'on en croit la lettre, reproduite par J. Lassalle, que lui aurait adressée F. Bayrou:

Jean Lassalle n'est pas un homme des temps modernes, pas un homme des temps politiques, où il s'agit de ruser, de se soumettre, dans un parti ou dans un courant, d'avaler toutes les couleuvres pour faire carrière. C'est un homme des temps héroïques, quand les êtres d'exception sont l'ordinaire de l'histoire, quand des êtres extraordinaires, qui sortent de l'ordinaire, font, parfois seuls contre tous, l'histoire ordinaire des hommes. (Lettre citée dans ALRF, p.75-76)

Certes, ici, J. Lassalle est indubitablement un homme. Néanmoins, la référence à des groupes sociaux - la paysannerie, les classes populaires, les travailleurs manuels, etc. - est moins directe et explicitée. L'ethos produit ici singularise indubitablement J. Lassalle. Il en fait un individu aux propriétés et à la personnalité remarquables. 


\section{Une individualisation politique particulière}

Le processus de singularisation associé au développement de l'ego-politique donne «à voir un individu doté d'une consistance identitaire qui l'émancipe de la stricte représentativité sociologique et politique » (Le Bart, 2013, p. 105). Ici, ce processus est notamment à l'œuvre dans la mise en avant d'un ethos viril de combattant en politique. Il n'est pas non plus totalement absent des récits produisant une relative identification aux groupes des paysans et des représentés. Néanmoins, la production de l'ethos de J. Lassalle se caractérise très fréquemment par des références claires aux groupes sociaux dont il entend être le représentant. L'ethos politique de J. Lassalle est donc construit à partir d'appartenances sociales (populaire et agricole), territoriales (rurale, montagnarde et béarnaise), sexuelles (masculine et hétérosexuelle²) et politiques (élu en rupture avec une classe politique technocratisée). La production de cet ethos montre la relation d'hétéronomie que les principes de légitimité du champ politique (au moins ceux prévalant dans ses régions relativement dominées) continuent à entretenir avec les principes de division du monde social, et cela malgré un processus général d’individualisation réel mais limité (Le Bart, 2008).

C'est bien parce que J. Lassalle a ces origines et ces qualités-là, et pas d'autres, qu'il se considère comme un représentant légitime non seulement des groupes sociaux auxquels il se réfère directement, mais aussi de l'ensemble du peuple (son appartenance à la tradition politique centriste l'invitant à se poser en rassembleur). L'étude de la production de son ethos à travers ses ouvrages politiques montre une individualisation réelle, mais plus partielle encore que celle des professionnels de la politique étudiés par C. Le Bart. Cette spécificité s'explique par une intégration relative à la classe politique nationale, intégration relative dont ses ouvrages politiques sont le reflet. Certes, J. Lassalle a une trajectoire faite de cumuls de mandats dans le temps et dans l'espace et profite d'une visibilité nationale. Néanmoins, il pâtit d'une pauvreté relative en diplômes et ressources partisanes et joue avant tout le rôle d'un acteur politique local au niveau national3. C'est donc en fonction de ces propriétés que J. Lassalle interprète un rôle politique - celui de publiant en politique -, qui n'est ni totalement subi, ni réellement choisi (Lagroye, 1997).

2. J. Lassalle ne manque pas de parler de son épouse et de ses enfants. Ses ouvrages sont encore l'occasion de commentaires valorisants pour le physique des femmes croisées, tour à tour «charmantes» (LPD, p. 271), «à la beauté radieuse » ( $L P D$, p. 210), « belle» (LRC, p. 232), J. Lassalle se décrivant comme n'étant «jamais en reste avec les jolies femmes» (LPD, p. 210). Ici aussi, J. Lassalle s'oppose à tout ce qui s'apparente à de la retenue excessive.

3. Ainsi, la probabilité du vote J. Lassalle à l'élection présidentielle de 2017 est clairement régionalisée; elle décroît fortement au fur et à mesure que l'on s'éloigne de Lourdios-Ichère et que l'on quitte les reliefs montagneux tout en se limitant avant tout aux pays de langue d'oc (Fourquet, 2017). 


\section{Références}

Amossy Ruth, 2014, "L'éthos et ses doubles contemporains. Perspectives disciplinaires », Langage et société, $\mathrm{n}^{\circ} 149$, p. 13-30.

- 2011, «Des sciences du langage aux sciences sociales : l'argumentation dans le discours", A contrario, n' ${ }^{16}$, p. 10-25.

Baudelot Christian, Establet Roger, 1992, Allez les filles !, Paris, Seuil.

BoltANSKI Laurent, ChIAPELLo Ève, 2011, Le nouvel esprit du capitalisme, Paris, Gallimard. Bourdieu Pierre, 2002, La domination masculine, Paris, Seuil.

- 2001a, Langage et pouvoir symbolique, Paris, Seuil.

- 2001b, Le bal des célibataires : crise de la société paysanne en Béarn, Paris, Seuil.

- 1981, «La représentation politique. Éléments pour une théorie du champ politique», Actes de la recherche en sciences sociales, $n^{0} 36-37, \mathrm{p} .3-24$

- 1980, Le sens pratique, Paris, Minuit.

Bourdieu Pierre, PASSERON Jean-Claude, 1964, Les héritiers : les étudiants et la culture, Paris, Minuit.

ChARAUdeau Patrick, 2005, Le discours politique : les masques du pouvoir, Paris, Vuibert.

Collovald Annie, 1989, "Les poujadistes ou l'échec en politique", Revue d'histoire moderne et contemporaine, $\mathrm{n}^{\circ} 36, \mathrm{p} .113-165$.

- 1988, "Identité(s) stratégique(s) », Actes de la recherche en sciences sociales, $\mathrm{n}^{\circ} 73$, p. 29-40.

CotTA Maurizio, Best Heinrich éd., 2007, Democratic Representation in Europe. Diversity, Change and Convergence, Oxford, Oxford University Press.

DuвoIs Vincent, Dulong Delphine éd., 1999, La question technocratique. De l'invention d'une figure aux transformations de l'action publique, Strasbourg, Presses universitaires de Strasbourg.

DuRu-Bellat Marie, Tenret Élise, 2009, «L'emprise de la méritocratie scolaire : quelle légitimité?", Revue française de sociologie, vol. L, n² 2, p. 229-258.

Elias Norbert, DunNing Éric, 1994, Sport et civilisation : la violence maîtrisée, Paris, Fayard.

FAURE Alain, NÉGRIER Emmanuel éd., 2017, La politique à l'épreuve des émotions, Rennes, Presses universitaires de Rennes.

FOURQUET Jérôme, 2017, «Jean Lassalle : un écho dans les montagnes des pays d'Oc ", dans Le vote disruptif: les élections présidentielles et législatives de 2017, P. Perrineau éd., Paris, Presses de Sciences Po, p. 245-250.

FUSULIER Bernard, 2011, «Le concept d'ethos. De ses usages classiques à un usage renouvelé », Recherches sociologiques et anthropologiques, vol. XLII, nº1, p. 97-109.

GAXIE Daniel, 2003, La démocratie représentative, Paris, Montchrestien.

- 1978, Le cens caché. Inégalités culturelles et ségrégation politique, Paris, Seuil.

GuIONNET Christine, 2002, «Entrées de femmes en politique. L'irréductibilité du genre à l'heure de la parité», Politix. Revue des sciences sociales du politique, n60, p.113-146.

Hervieu Bertrand, 2010, «Les agriculteurs dans la vie politique française. Cinquante ans d'évolution, quatre regards", dans Les mondes agricoles en politique : de la fin des paysans au retour de la question agricole, H. Bertrand, N. Mayer, P. Müller et al. éd., Paris, Presses de Sciences Po, p.19-38.

JOANA Jean, 1994, "Les usages de la méthode biographique en sciences sociales », Pôle Sud, nº 1, p. 89-99. 
KRIESI Hanspeter, 2014, «The populist challenge», West European Politics, vol. XXXVII, $n^{\circ} 2$, p. 361-378.

LAGRAVE Rose-Marie, 2009, «Filiations intellectuelles et espérance sociale. Figure et œuvre de Placide Rambaud », Études rurales, nº 183, p. 51-66.

LAGROYE Jacques, 2003, «Le leadership en questions. Configurations et formes de domination ", dans Le leadership politique et le territoire : les cadres d'analyse en débat, A. Smith et C. Sorbets éd., Rennes, Presses universitaires de Rennes, p. 47-69.

- 1997, "On ne subit pas son rôle », Politix. Revue des sciences sociales du politique, $\mathrm{n}^{\circ} 38, \mathrm{p} .7-17$.

LE BART Christian, 2013, L'ego-politique. Essai sur l'individualisation du champ politique, Paris, Armand Colin.

- 2012, La politique en librairie. Les stratégies de publication des professionnels de la politique, Paris, Armand Colin.

- 2008, L'individualisation, Paris, Presses de Sciences Po.

- 2003a, «L'analyse du discours politique : de la théorie des champs à la sociologie de la grandeur », Mots. Les langages du politique, nº 72, p. 97-110.

- 2003b, "La nostalgie chevaleresque chez les professionnels de la politique », dans Norbert Elias et la théorie de la civilisation, Y. Bonny, J.-M. de Queiroz et É. Neveu éd., Rennes, Presses universitaires de Rennes, p.169-183.

- 1998a, "L'écriture comme modalité d'exercice du métier politique », Revue française de science politique, vol. XLVIII, nº 1, p.76-96.

- 1998b, Le discours politique, Paris, PUF.

Le BART Christian, LefebVRe Rémi éd., 2005, La proximité en politique : usages, rhétoriques, pratiques, Rennes, Presses universitaires de Rennes.

LefebVRe Rémi, 2004, "La difficile notabilisation de Martine Aubry à Lille. Entre prescriptions de rôles et contraintes d'identité », Politix. Revue des sciences sociales du politique, n65, p. 119-146.

Leroux Pierre, RIUtoRt Philippe, 2013, La politique sur un plateau. Ce que la télévision fait à la représentation, Paris, PUF.

MazeAud Alice éd., 2014, Pratiques de la représentation politique, Rennes, Presses universitaires de Rennes.

NAUDET Jules, 2012, «Mobilité sociale et explications de la réussite en France, aux ÉtatsUnis et en Inde », Sociologie, vol. III, nº 1, p. 39-59.

NeVEu Érik, 1992, "Le sceptre, les masques et la plume», Mots. Les langages du politique, $\mathrm{n}^{0} 32, \mathrm{p} .7-27$.

NYE Robert A., 1994, «De l'honneur nobiliaire à l'honorabilité bourgeoise. Les origines de la masculinité moderne », Actes de la recherche en sciences sociales, nº 105, p. 45-61.

OfFERLÉ Michel éd., 2017, La profession politique, XIXe-xxıe siècles, Paris, Belin.

PUDAL Bernard, 1989, Prendre parti : pour une sociologie historique du PCF, Paris, Presses de la Fondation nationale des sciences politiques.

Rouban Luc, 2011, Les députés [numéro thématique], Les Cahiers du Cevipof, nº 55 .

SAWICKI Frédéric, 1994, "Laurent Fabius: du "Giscard de gauche” au "socialiste moderne". Analyse de la formation d'une identité politique», Pôle Sud, nº 1, p. 35-60.

SINGLY François de, 2013, «Le masculin pluriel», Travail, genre et sociétés, n² 29, p.161168.

TABET Paola, 1979, «Les mains, les outils, les armes », L'Homme, t. XIX, n³ 3-4, p. 5-61.

Weber Max, 1995, Économie et société. Tome I : Les catégories de la sociologie, Paris, Pocket. 
ZARCA Bernard, 1993, "L'artisanat. La plus populaire des classes moyennes? », Vingtième siècle. Revue d'histoire, 1993, n³ 37, p. 55-68.

- 1988, "Identité de métier et identité artisanale », Revue française de sociologie, vol. XXIX, nº 2 , p. 247-273.

\section{Résumé / Abstract / Compendio}

\section{L'ethos politique de Jean Lassalle à travers ses livres}

Cet article s'intéresse à la production de l'ethos de Jean Lassalle à travers ses livres politiques. L'étude de cette présentation de soi montre une variation autour du processus d'individualisation de la vie politique. Si cet homme politique reprend bien ce registre de légitimation, l'image qu'il donne à voir de sa personne dans ses ouvrages politiques reste particulièrement attachée aux groupes sociaux qu'il entend représenter en politique. Cette spécificité est à mettre en lien avec les propriétés particulières de cet acteur politique.

Mots-clés : ethos, individualisation, livres politiques, métier politique, représentation politique

\section{Jean Lassalle's ethos through his political books. Between political indivi- dualization and social representation}

This article focuses on the production of Jean Lassalle's ethos through his political books. The study of this presentation of self shows a variation on the individualization process of political life. This politician takes up this register of legitimation, but the image given in his political books remains strongly linked with the social groups which he intends to represent in politics. This specificity is explained by J. Lassalle's particular social characteristics.

Keywords: ethos, individualization, political books, political profession, political representation

\section{El ethos político de Jean Lassalle a través de sus libros. Entre la individualiza- ción política y la representación social}

Este artículo trata sobre la producción del ethos de Jean Lassalle a través de sus libros políticos. El estudio de este tipo de presentación de su propia persona constituye una variación acerca del proceso de individualización de la vida política. Si este hombre político integra bien esta modalidad de legitimación, la imagen que propone de su persona en sus libros políticos arraiga particularmente en los grupos sociales que pretende representar en la política. Se debe relacionar esta especificidad con las características de este actor político.

Palabras claves: ethos, individualización, libros políticos, profesión política, representación política 